\title{
$\mathrm{Pd}_{77.5} \mathrm{Au}_{6} \mathrm{Si}_{16.5}$ 合金无容器凝固 组织中的分形结构
}

\author{
孙力玲 (1) 吴 奇(12) 王文魁 ${ }^{(1)(3)}$ \\ (1) 中国科学院物理研究所、北京 100080; (2) 沈阳工业高等专科学校、沈阴 110044; (3) 中国科学院国际 \\ 材料物理中心, 沈阳 110015)
}

\section{关键词 $\mathrm{Pd}_{n .5} \mathrm{Au}_{6} \mathrm{Si}_{16.5}$ 合金 无容器疑固 分形}

合金凝固组织形态及其演化一直是现代凝固组织的核心问题. 近年来, 分形几何的发展 和应用为合金凝固组织的研究提供了一种新方法, 并取得了一定进展 $\left.{ }^{[1} \sim 3\right]$. 本文研究的 $\mathrm{Pd}_{n .5} \mathrm{Au}_{6} \mathrm{Si}_{16.5}$ 合金在无容器凝固环境下的凝固过程为一非平衡凝固过程, 其凝固组织中初生相 具有不规则的几何形貌, 用分形分析的方法来定量地描述其形貌及演变过程尚末见报道.

\section{1 实验方法}

试验合金的化学成分 (原子分数) 为 $\mathrm{Pd}_{7.5}, \mathrm{Au}_{6}, \mathrm{Si}_{16.5}$. 首先把熔炼好的试验合金置于 $1.2 \mathrm{~m}$ 落管装置顶部的石英玻璃坩锅中, 将落管抽真空至 $10^{-3} \mathrm{~Pa}$ 后, 通过电阻方法加热试样. 待样 品熔化后, 用氦气吹落合金液滴, 使其在自由下落过程中实现无容器凝固. 采用扫描电子显 微镜观察合金凝固组织中初生相形貌. 采用微区 X 射线衍射仪分析相结构. 采用图相分析 仪测定初生相的分形维数.

\section{2 实验结果}

图 1 为不同直径合金小球凝固组织中初生相形貌的背散射电子象. 可见, 当合金小球的 直径约为 $2.3 \mathrm{~mm}$ 时, 初生相的二维形貌呈仅具有一级分枝的枝杆状, 枝杆粗大, 二次臂枝芽 刚刚形成. 合金小球直径减小到约 $1.2 \mathrm{~mm}$ 时, 初生相形貌为二次臂发达的枝叶状. 当合金 小球的直径进一步减小到 $0.4 \mathrm{~mm}$ 时, 初生相形貌则演变成具有三级分枝的枝簇状. 研究表 明 ${ }^{[4]}$, 这种具有较强各向异性的树枝晶结构也具有标度不变性, 可以被看做是一种分形结构. 采用图象分析仪对上述不同直径合金小球凝固组织中初生相形貌的分形维数进行了, 测量, 测 量原理基于如下 ${ }^{\text {' }}$ :

$$
N=\mathrm{Fr}^{-D},
$$

其中, $N$ 为覆盖初生相的格子数, $F$ 为系数, $r$ 为每一个小方格子占原始格子的倍数, $D$ 为分形 维数. 测量结果表明, 当合金小球的直径为 $2.3 \mathrm{~mm}, 1.2 \mathrm{~mm}$ 和 $0.4 \mathrm{~mm}$ 时, 其初生相的分形维 数分别为 $1.14,1.18$ 和 1.22 (精度为 0.02 ). 图 2 为覆盖初生相的总格子数 $N$ 和测量小格子倍

1995-01-26 收稿, 1995-04-27 收侈改稿 


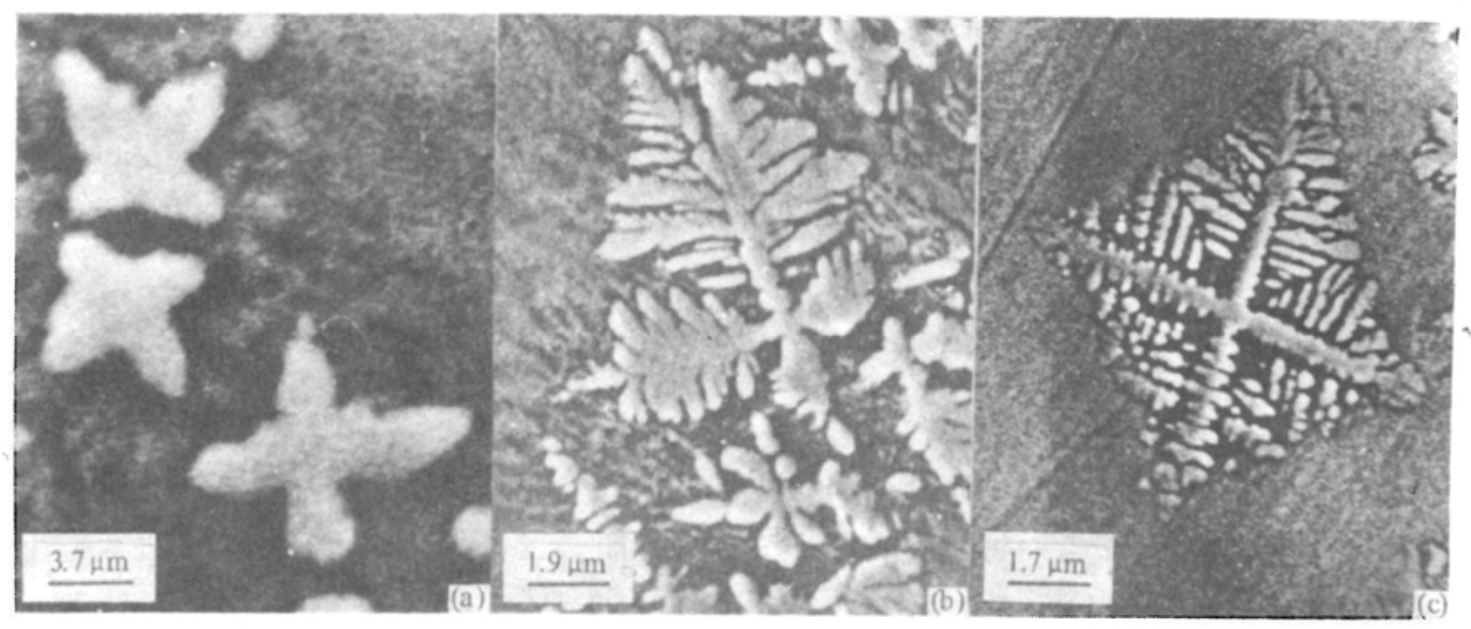

图 1 不同直径合金小球无容器凝固组织中初生相形貌的背散射电子象 (a) $d=2.3 \mathrm{~mm}$, (b) $d=1.2 \mathrm{~mm}$, (c) $d=0.4 \mathrm{~mm}$

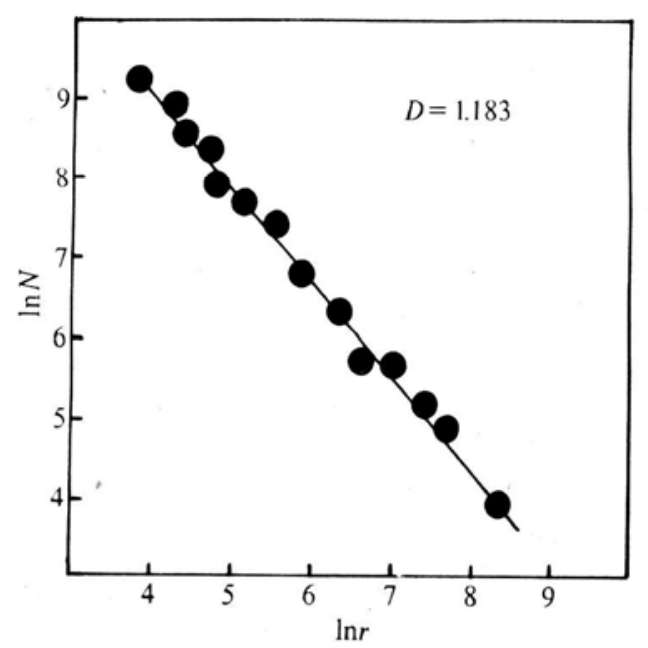

图 2 覆盖试验合金中初生相的格子数 $N$ 与每个小格子倍数 $r$ 之间的关系

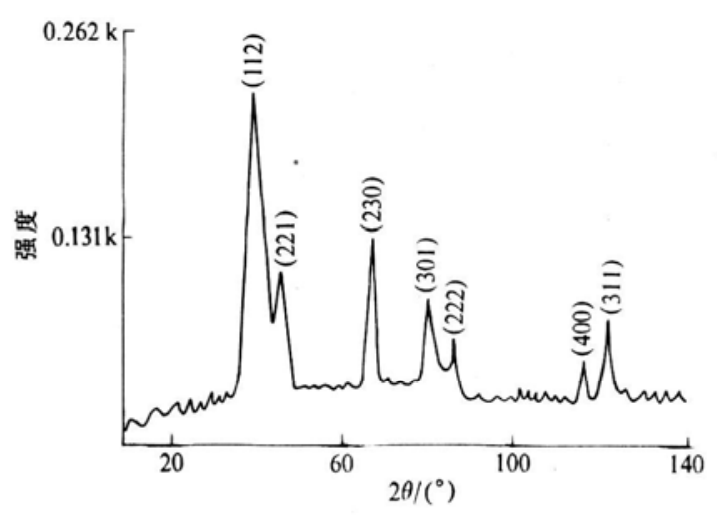

图 $3[\mathrm{Pd}, \mathrm{Au}]_{3} \mathrm{Si}$ 相 $\mathrm{X}$ 射线衍射谱图

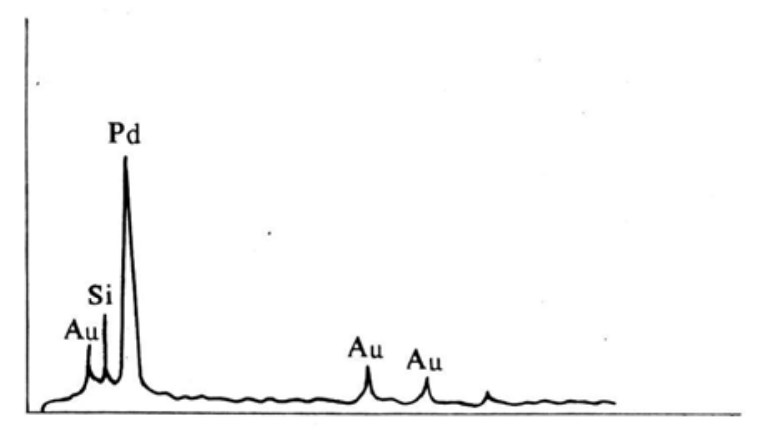

图 $4 \quad[\mathrm{Pd}, \mathrm{Au}]_{3} \mathrm{Si}$ 相能谱分析结果 
数 $r$ 的双对数图. 可见,在一定的码尺范围内, $\ln N$ 与 $\ln r$ 保持较好的线性关系,这表明被测初 生相的二维形貌确为一分形结构.

$\mathrm{X}$ 射线和能谱分析结果表明, 试验合金的无容器凝固组织中具有分形特征的初生相为 $[\mathbf{P d}$, $\mathrm{Au}]_{3} \mathrm{Si}$ 金属间化合物, 见图 3 和 4.

\section{3 分析与讨论}

分形结构产生的物理机制在于系统的耗散性 ${ }^{16}$. 试验合金在无容器凝固过程中,初生的 $[\mathrm{Pd}$, $\mathrm{Au}]_{3} \mathrm{Si}$ 相晶核一旦形成, 其生长界面前沿具有若干层原子宽度的区域便与其周围生长环境构 成了一种耗散系统, 它满足形成耗散结构的充要条件 而. 第一, 它是一个开放系统, 凝固过程 中初生的 $(\mathrm{Pd}, \mathrm{Au})_{3} \mathrm{Si}$ 相将不断地与外界 (液相) 进行能量和物质交换 (传热、传质过程). 第 二, 其生长过程远离平衡状态, 因为落管实验中合金小球的凝固过程是在很大的过冷度下进 行的 ${ }^{18}$. 第三, 系统中存在着非平衡涨落, 比如, 当合金小球直径变化引起的初生相生长界面 前沿的温度梯度和浓度梯度的变化. 第四, 系统要素之间具有非线性关系, 非平衡凝固条件 下温度和浓度的变化是非常复杂的, 这些影响因素之间的关系都是非线性的.

耗散结构具有自组织特性 ${ }^{[9]}$, 即当外界控制参数发生变化时, 系统会自发地调整与外界环 境物质, 能量的交换方式及速率来改变其生长形态. 对本研究来说, 合金小球直径的减小意 味着冷却速率增加, 初生的 $[\mathrm{Pd}, \mathrm{Au}]_{3} \mathrm{Si}$ 相与周围环境的传热、传质速率和强度加快, 最终导致 其形貌由仅具有一级分枝的枝杆状向具有三级分枝的枝簇状演化, 分形维数增大. 可见, [Pd, $\mathrm{Au}]_{3} \mathrm{Si}$ 相的生长过程是一个动态的自组织过程, 凝固组织中的每个分形花样均为系统耗散性 的几何表现. 关于合金凝固组织中这种耗散结构更深层次的分析还有待于深人研究.

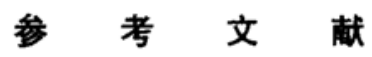

1 Hornbogen E. Fractals in microstructure of metals. Inter Mat Rev, 1989, 34(6): $277 \sim 296$

2 Dasher R F, Kessler D A, Levine $\mathrm{H}$ et al. The geometrical model of dendritic growth. Phys D, 1986, 21: 371 380

3 Sun Liling, Dong Lianke, Hu Zhuangqi. Fractal analysis of directional solidification behavior of Ni-base superalloy. Acta Metallurgica Sinica, 1993, 6A(5): $291 \sim 294$

4 Nittmann J, Stanley H E. Tip splitting without interfacial tension and dendritic growth pattern arising from molecular anisotropy. Nature, 1986, 321: 663

5 Tanaka Manabu, Lizuka Hiroshi. Charactarization of grain boundaries by fratal geometry and greep rupture properties of heat-resistent alloys. Z Metallde, 1991, 82:441 447

6 黄连科. 分形理论及其应用. 沈阳: 辽宁科学出版社, 1991. 24

7 Prigogine I. Introduction to Thermodynamics of Irreversible Processes, 3rd ed. New York: Interscience Pub, 1967. 88

8 许应凡, 王文鬿. 无容器过程中 $\mathrm{Pd}-\mathrm{Ni}-\mathrm{P}$ 系合金的过冷及金属玻璃的形成. 物理学报, 1990, 39(5): $836 \sim 842$

9 李如生. 非平衡态热力学和耗散结构. 北京: 清华大学出版社, 1986. 44 